\title{
Bilateral Primary Fallopian Tube Adenocarcinoma - A Rare Case
}

\author{
Bhavani C', Babu PVR ${ }^{2}$, Reddy ES ${ }^{3}$, Neeraja $\mathbf{M}^{4}$ \\ ${ }^{1}$ Dr C Bhavani, ${ }^{2}$ Dr P.V. Ramana Babu, ${ }^{3}$ Dr E. Sudhakar Reddy, ${ }^{4}$ Dr M. Neeraja, All affiliated to Dr. NTR University of \\ Health sciences, Vijayawada, Andhra Pradesh, India.
}

\begin{abstract}
Although fallopian tubes are a common site of metastases, primary fallopian tube carcinoma is considered a rare disease and is often mistaken histologically and clinically for ovarian cancer. The etiology is poorly understood and presenting symptoms are variable and non-specific, with preoperative diagnosis rarely entertained. We put forth our experience of a case of bilateral primary fallopian tube carcinoma which was histopathologically diagnosed after being misinterpreted on ultrasonography.
\end{abstract}

Key Words: Primary Fallopian Tube Carcinoma, Total Abdominal Hysterectomy, Bilateral Salpingo-oophorectomy

\section{Introduction}

Primary fallopian tube malignancy is the least common of all gynecological malignancies. It accounts for approximately $0.14-1.8 \%$ of all female genital tract malignancies [1]. The earliest description of this entity was given by Renaud in 1847 and the first microscopic description was recorded by Rokitansky in 1861 .

Since then, over 2000 cases have been reported in the literature [2]. The true incidence of primary fallopian tube carcinoma (PFTC) has been underestimated because of its close histological resemblance to ovarian cancer.

PFTC more frequently occurs between the fourth and sixth decades of life, with a median age of 55 years [3]. When compared to the secondary deposits which are often more common, single and localized, primary PFTC has been known to occur even bilaterally and with extensive intraperitoneal involvement. The vast majority $(>95 \%)$ of fallopian tube cancers are papillary serous and adenocarcinomas.

We report of a case of bilateral fallopian tube serous adenocarcinoma in a 68 year old female with emphasis on the diagnostic importance of post-surgical biopsy.

\section{Case Report}

A 68 year old postmenopausal female presented to the gynecological OPD with complaints of lower abdominal pain of 3 months duration with loss of appetite. No

Manuscript received: $28^{\text {th }}$ June 2014

Reviewed: $10^{\text {th }}$ July 2014

Author Corrected: $24^{\text {th }}$ July 2014

Accepted for Publication: $30^{\text {st }}$ July 2014 significant past history, family history was provided. General clinical examination revealed pallor with no significant findings per abdomen. Per vaginal examination revealed adnexal masses in both the lateral fornices. All her routine investigations were normal except for $\mathrm{Hb} \%$ which stood at $9.5 \mathrm{gm} \%$. Transabdominal ultrasound demonstrated echogenic and enlarged tubes bilaterally. A provisional diagnosis of bilateral hydrosalpinx was given. The patient underwent total abdominal hysterectomy with bilateral salpingooophorectomy and the specimen was sent for histopathological examination.

Because of the lack of surgical retroperitoneal assessment for lymph nodes FIGO staging universally followed has not been provided for our patient. A provisional stage of stage IB was provided. Post operative CT was called for and it revealed no abnormality. The cafeteria approach of treatment modalities were placed before our patient during follow up and as the patient was in stage IB, no neoadjuvant chemotherapy was rendered. The patient was followed up for 2 years and no recurrences were recorded so far.

\section{Gross Pathological Findings}

The uterus was slightly atrophic, measuring $8 \times 4 \times 2 \mathrm{cms}$ and weighed 30 gms. Bilateral symmetrical dilatation towards the fimbrial ends of the tubes was noted. External surface of the tubes was smooth and congested. Cut section of both the tubes revealed a grey white, solid friable growth within the lumen. Both the ovaries and the rest of the uterus were unremarkable. Image enclosed (Fig 1) 


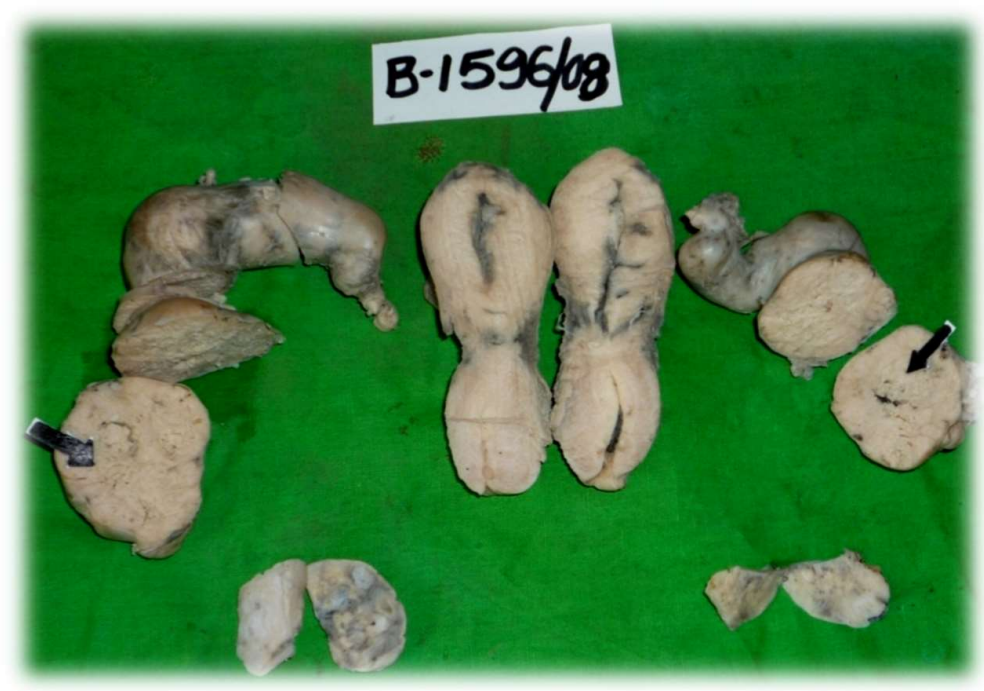

Fig.1: Gross photograph of the surgical specimen showing grey white tumour within the tubal lumen (marked by arrows).

\section{Histopathological Examination}

Sections from both the tubes revealed papillary serous adenocarcinoma extending from the lumen upto muscularis. Sections studied from the cervix, uterus and ovaries revealed no abnormality (Fig 2).

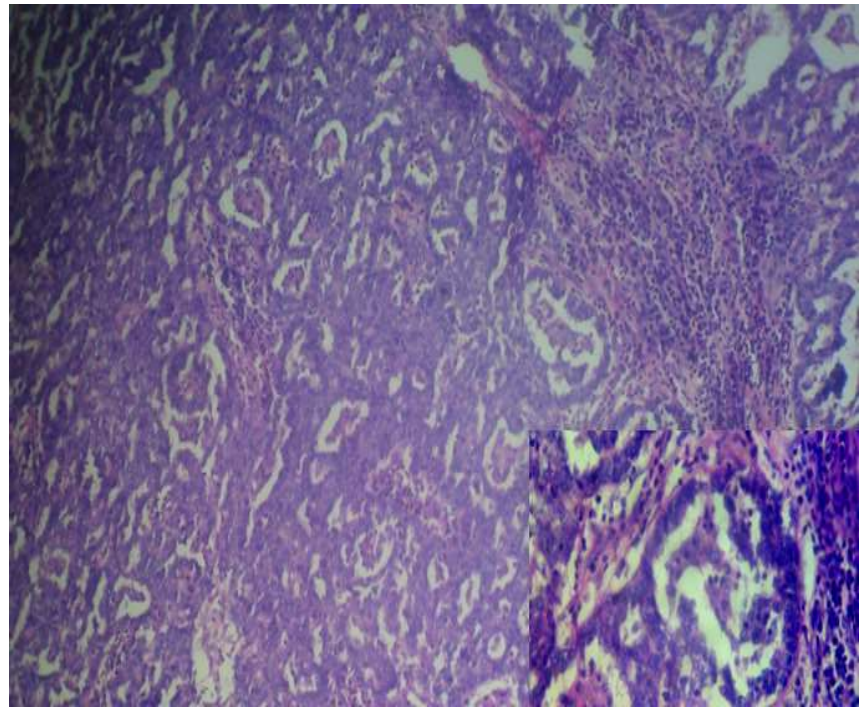

Fig 2: Microphotograph showing features of papillary serous adenocarcinoma with adjacent lymphoid infiltrate, $\mathrm{H} \& \mathrm{E}, 100 \mathrm{x}$ (400x within the inset ).

\section{Discussion}

Of about 2000 cases of primary fallopian tube carcinoma reported so far, only $20-25 \%$ of the cases are bilateral. Because of this rarity, we put forward our case for discussion.

Etiology of PFTC is unknown. Hormonal, reproductive and genetic factors also have been implicated. There is a Linkage with BRCA1 \& BRCA2 and other malignancies of ovaries, breast etc calls for evaluation of the family history in these cases [4]. PFTC is characterized by an extremely unstable phenotype and highly scattered DNA ploidy patterns and frequent p53 gene alterations.

Most of the tumours are usually asymptomatic and so preoperative diagnosis stands only in the range of $0 \%$ - 
$10 \%$. Latzko's triad of symptoms consisting of intermittent profuse serosanguineous vaginal discharge, colicky abdominal pain relieved by discharge and abdominal or pelvic mass have been reported in $15 \%$ of cases. Hydrops tubae profluens implying intermittent discharge of clear or blood tinged fluid spontaneously or on pressure followed by shrinkage of adnexal mass occurs in only $5 \%$ of patients [2].

The echographic appearance of fallopian tubes in PFTC is nonspecific, mimicking other pelvic diseases such as tuboovarian abscess, ectopic pregnancy and ovarian tumour. Transvaginal ultrasound may provide a better picture over transabdominal ultrasound as has been described by Timor-Tritsch and Rottem [5].

Three dimensional colour Doppler when combined with transvaginal ultrasound can aid in detection of neovascularization and papillary patterns characteristic of PFTC [6].

Pap smear positivity occurs in only $10-36 \%$ of cases. Our case did not show any significant findings in this regard. In conjunction with this diagnosis may be suspected in cases of postmenopausal bleeding with negative findings on colposcopy, cervical biopsy and diagnostic curettage.

CA-125 was found to be a better prognostic marker and its elevation in serum was found to be non diagnostic given its spectrum. Meng et al reported that even an intraoperative diagnosis is missed in upto $50 \%$ of patients [7].

The pathological diagnostic criteria of PFTC first put forth by $\mathrm{Hu}$ and his colleagues which were later modified slightly by Sidles were fulfilled in our case. Accordingly: (a) grossly, the main tumour was in the tube and was seen arising from the endosalpinx; (b) histologically the pattern reproduced the epithelium of the mucosa and showed a papillary pattern; (c) transition from benign to malignant tubal epithelium was demonstrated; and (d) the ovaries and endometrium were normal.

Penetration of the serosa, a poor prognostic factor was not seen in our case.

Surgery remains the mainstay of treatment with TAH \& BSO and a thorough retroperitoneal assessment. Adjuvant chemotherapy with platinum based regimen and hormonotherapy have been increasingly advocated in advanced disease [8].Generally the reported 5- year survival rate is about $65 \%$ or higher [9].
The stage of disease at the time of diagnosis is the most important prognostic factor in this regard.

\section{Conclusion}

PFTC is a rare tumour accounting for $<1 \%$ of all female genital tract cancers. A potential biomarker for early detection of PFTC is awaited in this era of molecular biology given the limitation of "Man behind the machine" in radiology.

The data regarding the treatment of fallopian tube cancers are limited and extrapolated from the ovarian cancer literature. A professional dialogue and exchange of pathological material may aid in scientific research to unravel the mysteries behind PFTC.

\section{Funding: Nil}

\section{Conflict of interest: None}

Permission from IRB: Yes

\section{References}

1. Riska A, Leminen A, Pukkala E. Sociodemographic determinants of incidence of primary fallopian tube carcinoma,Finland 1953-97. Int $J$ Cancer.2003;104 (5):643-645.

2. Dimitrios Pectasides, Eirini Pectasides and Theofanis Economopoulos. Fallopian Tube Carcinoma: A Review. The Oncologist.2006;11 (8):902-912.

3. Boutselis JG, Thompson JN.. Clinical aspects of primary carcinoma of the Fallopian tube: a clinical study of 14 cases.Am J Obstet Gynecol .1971;111(1):98-101.

4. Jeung IC, Lee YS, Lee HN, Park EK. Primary carcinoma of the fallopian tube: report of two cases with literature review. Cancer Res Treat.2009;41(2):113-116.

5. Timor-Tritsch IE, Rottem S. Transvaginal ultrasonographic study of the fallopian tube. Obstet Gynecol .1987;70(3):424-428.

6. Kurjak A, Kupesic S, Sparac V. Kosuta D. Threedimensional ultrasonographic and power Doppler characterization of ovarian lesions.. Ultrasound Obstet Gynecol .2000;16(4):365-371.

7. Kalyani R, Kumar ML, Srikantia SH. Primary adenocarcinoma of fallopian tube - a case report. Indian J Pathol Microbiol. 2005;48(2):219-21. 
8. Meng ML, Gan-Gao, Scheng-Sun. Bao Qin Chou, Jung Ziang. Diagnosis of primary adenocarcinoma of the fallopian tube. $\mathrm{J}$ Cancer Res Clin Oncol 1985;110(2):136-140.
9. Inal MM, Hanhan M, Pilanci B.Tinar S. Fallopian tube malignancies: experience of Social Security Agency Aegean Maternity Hospital. Int J Gynecol Cancer 2004;14(4):595-599.

\section{How to cite this article?}

Bhavani C, Babu PVR, Reddy ES, Neeraja M. Bilateral Primary Fallopian Tube Adenocarcinoma - A Rare Case. Int J Med Res Rev 2014;2(4):393-396. doi:10.17511/ijmrr.2014.i04.22 